\title{
Data Acquisition System for Highway Engineering Construction Safety Based on Android Platform
}

\author{
Linliang Zhang ${ }^{1, a^{*}}$, Lei Jia ${ }^{2, b}$, and Zhiying Liu ${ }^{2, b}$ \\ ${ }^{1}$ Shanxi Transportation Research Institute, XueFu Street $79^{\text {th }}$, Taiyuan City, Shanxi Province, \\ China \\ azhanglinliang@163.com
}

Keywords: Android; Mobile Client; Data Acquisition; Safety

\begin{abstract}
Aiming at the difficulties such as low efficiency of data collection, large amount of data and much hidden danger and risk identification in process of highway engineering construction safety and evaluation, this paper proposes a data acquisition system for highway engineering construction safety based on Android platform. This system is aimed at improving the data acquisition efficiency, avoiding data sorting and computational complexity, and realizing automatic construction safety evaluation. And through the wireless network, the system completes communication and data transmission with Web Service, thus to realize remote data acquisition; and uses the Android Client and Web Client customizable interface to display data, so the system has good robustness and intelligence.
\end{abstract}

\section{Introduction}

The importance of highway engineering construction safety is self-evident, which directly affects people's life and property safety. And the acquisition of hidden danger data and dangerous source data during highway engineering construction is a prerequisite for construction safety evaluation. However, highway engineering construction safety data is mostly collected in the traditional way - remembering hidden danger and dangerous sources by brain, and then recording in the field by hand. but because hidden danger sources and dangerous sources are involved in the point, line, and surface aspects, moreover, single items and sub-items for inspection in hidden danger are numerous, it's difficult to remember, easy to forget and miss notes; after manual records, need to classify hidden data and risk data in detail according to individual and sub-items, which brings complexity, low efficiency and statistical difficulties, thus it is more difficult to achieve safety evaluation for highway engineering construction. Although efficient to enter the input through the Web way, it's often confined to network. To achieve limited network connection in highway construction, it is difficult to meet the actual work needs, and cannot achieve all the highway location data acquisition, such as tunnel excavation, distribution room, pump room and other locations. Therefore, the current manual recording or web way for highway construction safety data acquisition has been unable to complete the requirements of on-site safety data acquisition.

Android system is a software platform and operating system based on the Linux kernel, which uses software-stack architecture, low-level taking Linux kernel work as the basis, to provide basic functions developed by Google; other applications developed by other companies, which have worked with dozens of mobile phone companies to establish a development alliance. Now Android system or platform is widely used in mobile devices, PC, workstations and Internet TV, etc., moreover, the portability of Android mobile terminal allows it to be applied in specific occasions and environment, so as to complete the work needs and operations under specific conditions [1].

According to the actual demand and work content of highway engineering, this paper proposes a mobile client data acquisition system for highway engineering construction safety based on Android. The system can realize customized UI design interface, and the Mobile Client App can communicate with the remote server (Web Service) for data communication, to realize automatic data request, user login verification, network environment detection, offline data local save, online data remote transmission and automatic data transmission and other functions to meet the job needs of different 
conditions from different users. According to different logic rules and operational content, this system will design different UI interfaces to complete different tasks.

\section{Overview and Framework Design}

According to the inspection and evaluation requirements of highway engineering construction safety (local standard), the main functions of Mobile Client include data acquisition module such as user login, hidden danger check, hidden danger rectification, hidden danger recheck, hidden danger acceptance, hidden danger spot check, rectification acceptance, risk source check. Each unit will be evaluated and rated for safety management according to the situation of inspection, rectification and acceptance of the hidden danger. Related highway engineering construction safety units include builders, supervision units, construction units and safety supervision units etc. In the process of highway engineering construction, it is a must work for all construction management units, supervision units and builders to collect the data of potential risks and dangerous sources. According to the requirements of national standards and local standards, the safety data collection of highway engineering construction needs to be designed logically and in detail according to the actual needs, including safety data types, definition mode, design approach, data request and transmission, data local-storage and remote data storage etc., at the same time to be carried on the statistics and sorted out to complete the entire business process according to the safety data types, and according to the situation of the inspection, spot checks of the hidden dangers from each unit, the data collection process of specific hidden danger inspection, rectification and acceptance is shown in Figure 1.

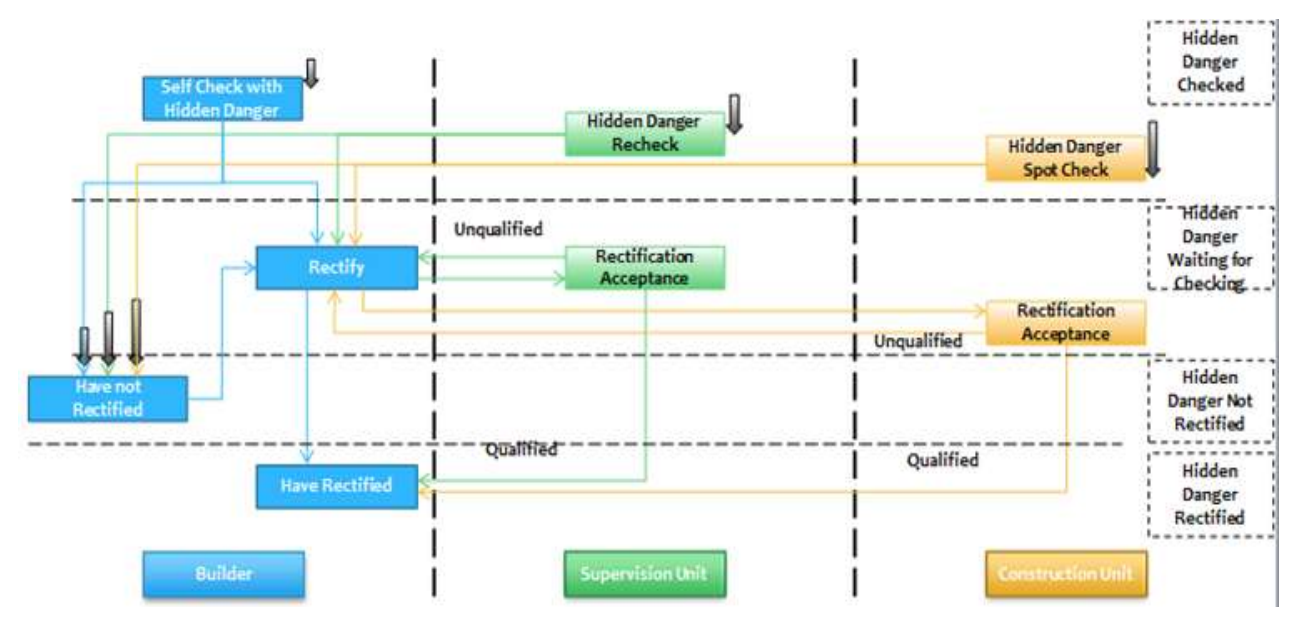

Figure 1. Data acquisition flow chart

The builder needs to conduct self-examination of the hidden danger in highway engineering construction, the hidden dangers checked should be rectified within required date, according to the property and different content of hidden dangers, the unit being inspected will be deducted points with weighing; hidden dangers rectified after rectification date will be conducted rectification acceptance, those that haven't been rectified will be continually penalized with weighing; the inspection unit needs to recheck the hidden danger checked by the builder, while checking those hidden dangers not checked by the builder, and penalize the builder for the hidden dangers checked and those reviewed but haven't been rectified, the point deduction of the supervision unit shall carry out on the basis of the builder's point deduction; and the construction unit shall make spot check on the inspection of the supervision unit and the builder, and deduct points with weighing to the builder and the inspection unit according to the hidden dangers checked, and ultimately calculate the score statements of each unit, including monthly statements, annual statements (Note: the longer the arrow the more points deducted). 


\section{Network Topology}

Android System Client carries data transmission mainly through the wireless network, by phone loaded with Android Mobile App Client or PC with remote Web Service to communicate, Web Service responsible for data transmission, data extraction, data validation and other work. Web Service and Database conduct data exchange, complete the data storage, and establish the basic data for highway engineering construction safety evaluation by formatting the stored data. The system network topology is shown in Figure 2 [2].

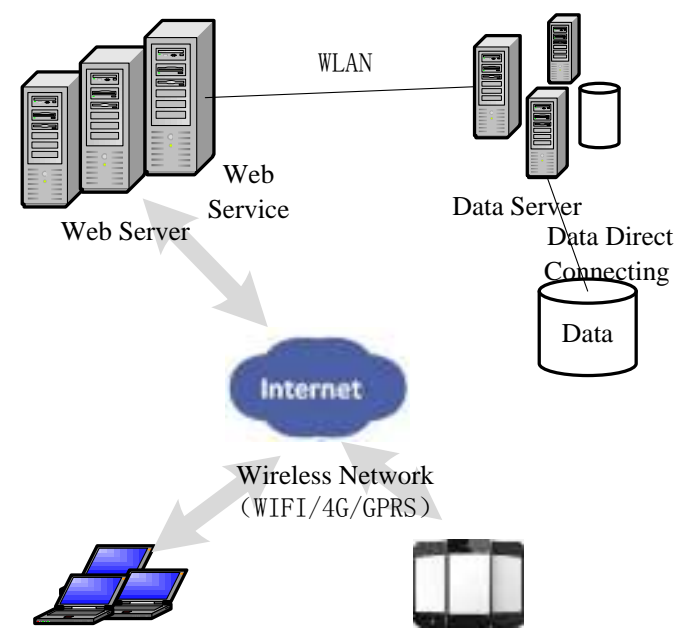

Figure 2. System topology diagram

\section{System Modular Design}

The system is mainly composed of three parts, dividing into Mobile App Client, Web Service and Database System; App Client mainly involves the interface display, user login and verification, data request and transmission, data local storage, network monitoring and WIFI automatic switching, offline data storage, image compression and packaging functions etc.,[3]; Web Service mainly includes receiving data requests, extract data, data conversion, data transmission, data interface definition and other functions; Database is composed of data storage and data analysis, trigger different data operations according to stored data, and sorts and stores collected safety data from different types of highway engineering construction. UML system data acquisition flow chart of this system is shown in Figure 3.

First, download the Mobile App Client, after successful installation, when the user logs in, conduct the user authentication, App Client achieves a simple local user authentication in the process of user authentication, Web Service achieves complex encryption user authentication, so as to realize the double-layer security password protection for users. After the user logs in, request data according to the login user information, connect the network with the remote Web Service, extract data according to the agreed rules, return the extracted data to the Client, and then parse data. The Client completes the interface display through loading the UI interface, parsing UI control and loading the request data; the user, according to the business logic, collects data, then saves data after completing data collection, through the network verification to achieve local storage of offline data and remote storage of wireless network data [4]. 


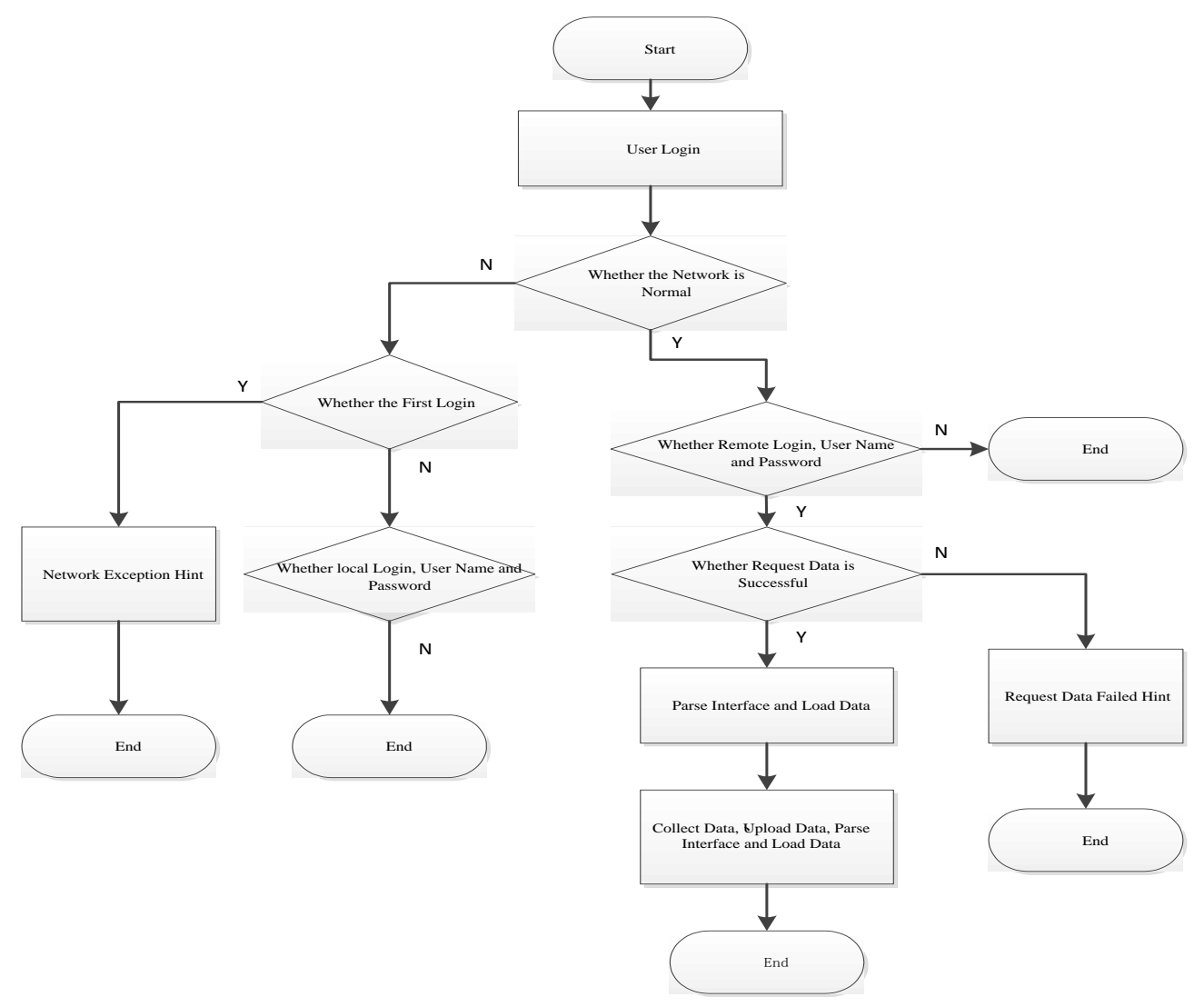

Figure 3. Data acquisition flow chart

\section{Key Technology Research}

The system consists of three parts, namely App Client, Web Service and Database, according to the special environment of highway engineering construction safety, some specific functions needs to be achieved, so the specific functions of each part need to be developed. Due to different resolution and screen sizes of smart phone and PC, need to design compatibility interface for the App Client. Display compatibility interface with different shapes according to different resolution and interface sizes, through different UI interface design, use script to develop compatibility interfaces; App Client monitors the status of wireless network, at the same time, automatically switches between networks according to different network status to realize the automatic switch between mobile network signal and WIFI signal; meanwhile according to network status, realize offline data storage, when the network is not signaled, the data is stored locally. Once the network signal is found to meet the data transmission requirements, transmit the data automatically and monitor the data integrity; the Web Service needs to verify the login user, encrypt the data, store the encryption key and the decryption key, and then sent the decryption key to the Client according to the user request, the Client analyzes the data according to the decryption key; the App Client can transmit the compressed picture through the Bluetooth function to the Web Client, and store into the server database through the Web Client .

\section{Data Transmission and UI Interface Display}

When the App Client requests data, need to verify user, during user authentication, Web Service through the asymmetric key algorithm generates an encryption key and a decryption key, through the encryption key to encrypt the password, transfer the decryption key to the Client to decode the password, which shows the password security guarantee. After successful user authentication, according to the Web Service data request interface, App Client makes data request, Web Service parses App Client request, and then requests data from the database, and converts the requested data into JSON packets 
for data transfer, App Client will also convert collected data into JSON for transmission in the same way. JSON (JavaScript Object Notation) is a lightweight data exchange format, compared to XML, TXT and other text, easy to parse, and is independent of language and platform, JSON is in accordance with the name / value pairs. The format is as follows:

\{"people":[\{"firstName":"Brett","lastName":"McLaughlin"\},

\{"firstName":"Jason","lastName":"Hunter" \}, ～～"firstName":"Elliotte","lastName":"Harold"\} ] \}

Web Service extracts data from the database, then converts into JSON data through DataSet, the Client will receive the JSON data, parse, and then according to different UI interface control needs, loads JSON data according to different fields. After the user finishes data collection, the interface UI control data will be converted into JSON data for data storage, through the network transmission to the Web Service and parse, saved to the database, data transmission is mainly done through the Ajax technology for asynchronous data transmission.

When it comes to picture upload, considering different resolution and pixels of different devices, pictures need to be compressed uniformly, through Bitmap image can be compressed by size or quality; you can also use localResizeIMG plug from Google, thus the picture will be converted to specified width and quality, then into base64 encoding, and then sent to the Web Service through Ajax, after the analysis of the Web Service finally be stored to the server specified directory, or be converted into binary encoding or bitmap and stored directly into the database.

\section{Network Monitoring and Data Storage}

When the data is collected by Mobile App Client, it is also very important to monitor the network signal, the 3G / 4G network and WIFI signal. When the intensity of WIFI signal is high, the locally saved data and pictures are automatically transmitted. When the WIFI signal is insufficient, the user will be prompted to whether transmit the data through the mobile traffic, thus to avoid traffic consumption [6], and the changes of WIFI signal icon and signal strength are completed by the System UI, in the directory

framework/base/packages/SystemUI/src/com/android/systemui/statusbar/policy/NetworkController. java

This class has implemented the Receiver for System UI to monitor network connection status.

According to the actual needs, when the monitoring network status is online and the signal strength is adequate, transmit remote data, save the data to the remote database for remote data storage; when the monitoring network status is offline or signal strength is inadequate, offline storage (local storage) will be conducted, and in the highway construction environment, the App Client needs to save the remote request data to the local database [7]. Android system uses an open source, operating system independent SQL database SQLite, which is a light-weighted database, complying with ACID relational database management system, very low occupation of resources; its design goal is mainly embedded. SQLite has become the complex data storage engine of Android Mobile device, generating the database and database tables through the code, and customizing database relational table [8] according to the needs.

\section{Application Examples}

According to the safety evaluation standard in one province and national standard of highway engineering construction, and the specific needs in the highway environment, the Mobile App Client with compatibility is designed according to the different screen and resolution, to realize the interface self-adaption, and applied to different sizes and different specifications of the mobile terminal. After landing success, the page will achieve the Layout way, and can automatically load the UI control and the requested data source. at the same time, the Client will automatically generate local database and database table, will sort and store the requested data source in the local database, and directly save after the completion of filling, through offline storage or remote storage for data save. 


\section{Conclusions}

In this paper, based on the Android system, the data acquisition system for highway engineering construction safety has been developed to solve all the problems of data collection of hidden danger and risk source facing traditional highway builders, supervision units and construction units, such as:

A. difficulty in remembering local standards and national standards, easy to forget or miss out;

B. avoid the complexity of data records and workload, so as to improve the work efficiency and reduce mistakes in work;

C. meet specific application requirements, save data whether online or connected.

\section{Acknowledgements}

《"Internet + Highway Engineering "Construction Safety Management and Inspection Evaluation Technnology Research》, Project No.: 2017-1-24, Research Project of Transport Department of Shanxi Province .

\section{References}

[1] Y.M. Yao, W.G. Liu. Study of Android's Architecture and Its Application Development[J]. COMPUTER SYSTEMS \& APPLICATIONS, 2008, 17(11):110-112.

[2] Jin Zhang, J.K. Yang and X.B. Hu. Field Quality Data Acquisition System Based on Android Platform[J]. COMPUTER SYSTEMS \& APPLICATIONS, 2016, 25(9):109-112.

[3] Wei Yan, J.K.Ye. The application of multithreading technology in android mobile development[J]. Informotion \& Communications, 2012(1):46-47.

[4] Ke Wang, HongBin Ma. Design and Implementation of a Data Acquisition System Based on the Android Platform[J]. Geomatics \& Spatial Information Technology, 2014(10):29-32.

[5] D.J.Geng, Yue Suo and Yu Chen etc. Design and implementation of Android phone based access and control in smart space[J]. JOURNAL OF COMPUTER APPLICATIONS, 2011, 31(2):559-561

[6] Lei Zeng, H.F.Zhang and W.Y.Hou. Design and Implement of WiFi Wireless Measurement and Control Network[J]. Electrical Measurement \& Instrumentation, 2011, 48(7):81-83.

[7] S.W.Zhou, W.B.Xie. Design and implementation of smart home terminal based on Android[J]. Microcomputer \& Its Applications, 2012,31(14):10-13.

[8] Y.M.Yao, W.G.Liu. Study of Android's Architecture and Its Application Development[J]. COMPUTER SYSTEMS \& APPLICATIONS, 2008, 17(11):110-112. 